\title{
MULTIPLE CONGENITAL MALFORMATIONS OF FEMALE INTERNAL GENITALIA: ITS SURGICAL CORRECTION
}

Ritu Mishraํㅜ Aditya Prakash Misra²

\section{HOW TO CITE THIS ARTICLE:}

Ritu Mishra, Aditya Prakash Misra. "Multiple Congenital Malformation of Female Internal Genitalia: Its Surgical Correction". Journal of Evolution of Medical and Dental Sciences 2014; Vol. 3, Issue 34, August 11;

Page: 9129-9132, DOI: $10.14260 /$ jemds/2014/3178

ABSTRACT: BACKGROUND: Rare case of the patient having multiple developmental variations of mullerian duct: unicornuate uterus with non-communicating rudimentary horn, cervical agenesis and transverse vaginal septum with cryptomenorrhoea. AIMS: Restoration of menstrual function and restoration of reproductive function. METHODS AND MATERIAL: Patient was examined and then surgical repair of reproductiove organs was done. RESULTS AND CONCLUSIONS: Patients responded well in post-operative period. She started having normal menses without any complaints. Patients with cryptomenorrhea must be thoroughly evaluated and appropriate surgical correction of underlying congenital anamoly provides good results.

KEYWORDS: Mullerian anomaly, Transverse vaginal septum, Cryptomenorrhoea, Metroplasty, Rail road repair.

INTRODUCTION: Mullerian duct developmental variations are estimated to occur in $0.1-0.5 \%$ of women. The true prevalence is unknown because the variants usually are discovered in patients presenting with infertility.

Two paired Mullerian ducts ultimately develop into the structures of the female reproductive tract. The structures include the fallopian tubes, uterus, cervix, and upper two thirds of the vagina. The ovaries and lower one third of the vagina have separate embryologic origins from genital ridge and urogenital sinus respectively, not derived from the Mullerian system. Any deviation at any point of development may lead to various malformations.

Certain types of the variations can increase morbidity, such as in patients with obstructed or partially obstructed mullerian systems, who present with hematosalphinx, hematocolpos, retrograde menses and endometriosis. Women of childbearing age often present with various problems of infertility, repeated spontaneous abortions, or premature delivery.

The variant is discovered incidentally during imaging evaluation for infertility workup or any other condition or during surgery. Sometimes surgical management is warranted for symptomatic cases in the form of resection of vaginal septum, vaginoplasty, cervical reconstruction, metroplasty or combination of any of these.

This is rare case in which the patient is having multiple developmental variations: unicornuate uterus with non-communicating rudimentary horn, cervical agenesis and transverse vaginal septum.

CASE REPORT: A 15 years old unmarried girl was admitted in our hospital with history of primary amenorrhea and cyclical lower abdominal pain since 1 year. Patient had history of exploratory laparotomy 2 years back for ovarian cyst. Her vitals were stable at the time of admission. On palpation of abdomen she had mild lower abdominal tenderness. According to history provisional 
diagnosis of cryptomenorrhoea was made. Examination under general anaesthesia revealed blind vaginal pouch, giving impression of high transverse vaginal septum. Surgery was planned for resection of vaginal septum and hematometra drainage.

All pre-operative investigations were normal. Ultrasonography revealed bicornuate uterus with hematometra with bilateral normal kidney.

Patient was taken for surgery. Under general anaesthesia resection of transverse vaginal septum was done but cervix could not be visualized or felt so decision of exploratory laparotomy was taken. Abdomen was opened by infraumblical right paramedian incision. Uterus was bulky, with rudimentary horn on right side which was connected to left horn of uterus through a fibrous band but non-communicating. Right adnexa attached to rudimentary horn was normal while on left horn of uterus dense adhesions were found and ovary, fallopian tube were absent (figure 1).

An Incision was given over anterior surface of enlarged left horn of uterus, tarry dark material escaped through the incision (figure 2). A Hegar's dilator was passed through uterus to check the communication of uterine cavity with vaginal canal but it was found that lower part of uterus was blind with no cervix. Using the dilator as a guide, a nick was given on lower blind part of uterus per vaginally at the tip of dilator to connect uterine cavity with vagina (Rail road repair) (figure 3).

As rudimentary horn could not be excised because adnexa attached to it was normal and on the other side it was absent so in view to restore normal reproductive functions, cavity of rudimentary horn was connected to uterine cavity (left horn) through metroplasty. Fibrous band between two horns was excised. Keeping in mind the method of strassman metroplasty, incision was given on medial wall of both rudimentary horn and left horn of uterus. Anterior wall and posterior wall of rudimentary horn was stitched to anterior and posterior wall of left horn of uterus respectively, by interrupted sutures in two layers (figure 4).

The incision on left horn through which Hegar's dilator was passed, was closed. Foley's catheter was placed in uterine cavity and bulb inflated with $20 \mathrm{cc}$ of normal saline so that uterine content can be drained out and patency of the tract can be maintained (figure 5). Hemostasis maintained and abdomen closed in layers. Abdominal and perineal dressing was done.

Patient's postoperative period was uneventful. She was discharged on $10^{\text {th }}$ post-operative day with intrauterine catheter in situ and was advised regular follow up every 15 days. Since then, patient is having normal menses and is having no complaints.

DISCUSSION: Mal development of the mullerian ducts occur in variety of forms and each variant is distinctive. American Fertility Society (AFS) categorized urogenital anomalies in 4 class:

- Class I dysgenesis of mullerian ducts.

- Class II disorders of vertical fusion of mullerian ducts

- Class III disorders of lateral fusion of mullerian ducts.

- Class IV unusual configurations of vertical lateral fusion defects.

Classifications of developmental variations of vagina based on certain anatomic findings are useful in organizing the type of malformation, but usually there are exceptions to each rule. Thus, what appeared, after preliminary diagnostic evaluation, to be an apparently isolated vaginal malformation i.e. transverse vaginal septum (AFS class II) and resection of septum was planned, but 


\section{CASE REPORT}

only after exploratory laparotomy it was found to be associated with a developmental variations of uterus and cervix. Finally it was found to be AFS class IV, having transverse vaginal septum and cervical agenesis as a part of vertical fusion defect and unicornuate uterus with non-communicating rudimentary horn with cavity as a part of lateral fusion defect.

A comprehensive pre-operative evaluation of patients with suspected malformations of the mullerian ducts is essential, but a clear understanding of the particular variant may not be established until the time of surgical correction. Dysmenorrhoea, abnormal and heavy menstrual bleeding have been reported to occur more frequently with any form of double uterus and to be relieved after unification operations. Capraro et al.[1]

Reported several cases in which dysmenorrhoea were cured by metroplasty. Patient with congenital absence of the cervix present a diagnostic challenge. Patients with cervical aplasia with a functional midline uterine corpus have aplasia of the lower two thirds of the vagina with an upper vaginal pouch. Valdes et al[2] have reported the use of pre-operative ultrasonography in evaluation of two patients with atresia of vagina and cervix but in our case these variants were not detected by ultrasonography. MRI has been found helpful in confirming the diagnosis as said by Markham et al.[3]

One of the two aims of the surgery i.e. restoration of menstrual function has been achieved and second one i.e. restoration of reproductive function has to be studied in follow up.

Artificial connection between two horns may become fibrosed. Abnormal uterine milieu, cervical agenesis itself or by abnormal semen deposition, associated endometriosis and some other etiological factors may lead to subfertility or infertility.

Cervical agenesis is the major limiting factor for establishment of reproductive function. Only occasional success in maintaining an open passageway and normal cyclic menstruation have been reported. If we will succeed in achieving pregnancy, risk of ectopic pregnancy especially rudimentary horn pregnancy, spontaneous abortion, cervical insufficiency, preterm labour, abnormal labour will always be there.

Regular and frequent antenatal visit, earliest possible ultrasonography for confirmation of intrauterine pregnancy with exclusion of rudimentary horn pregnancy, mandatory hospital delivery and elective caesarian section are the advice to be given regarding pregnancy. Rest of the management can be planned according to circumstances and available recent advances.

\section{REFERENCES:}

1. Capraro VJ, Gallago MB. Vaginal agenesis. Am. J Obstet Gynaecol 1976; 124: 98.

2. Valdes C, Malini S, Malinak L. Sonography in the surgical management of vaginal and cervical atresia. Feril Steril 1983; 40: 263.

3. Markham SM, Parmley TH, Murphy AA, et al. cervical agenesis combined with vaginal agenesis diagnosed by MRI. Fertil Steril 1987; 48:143. 


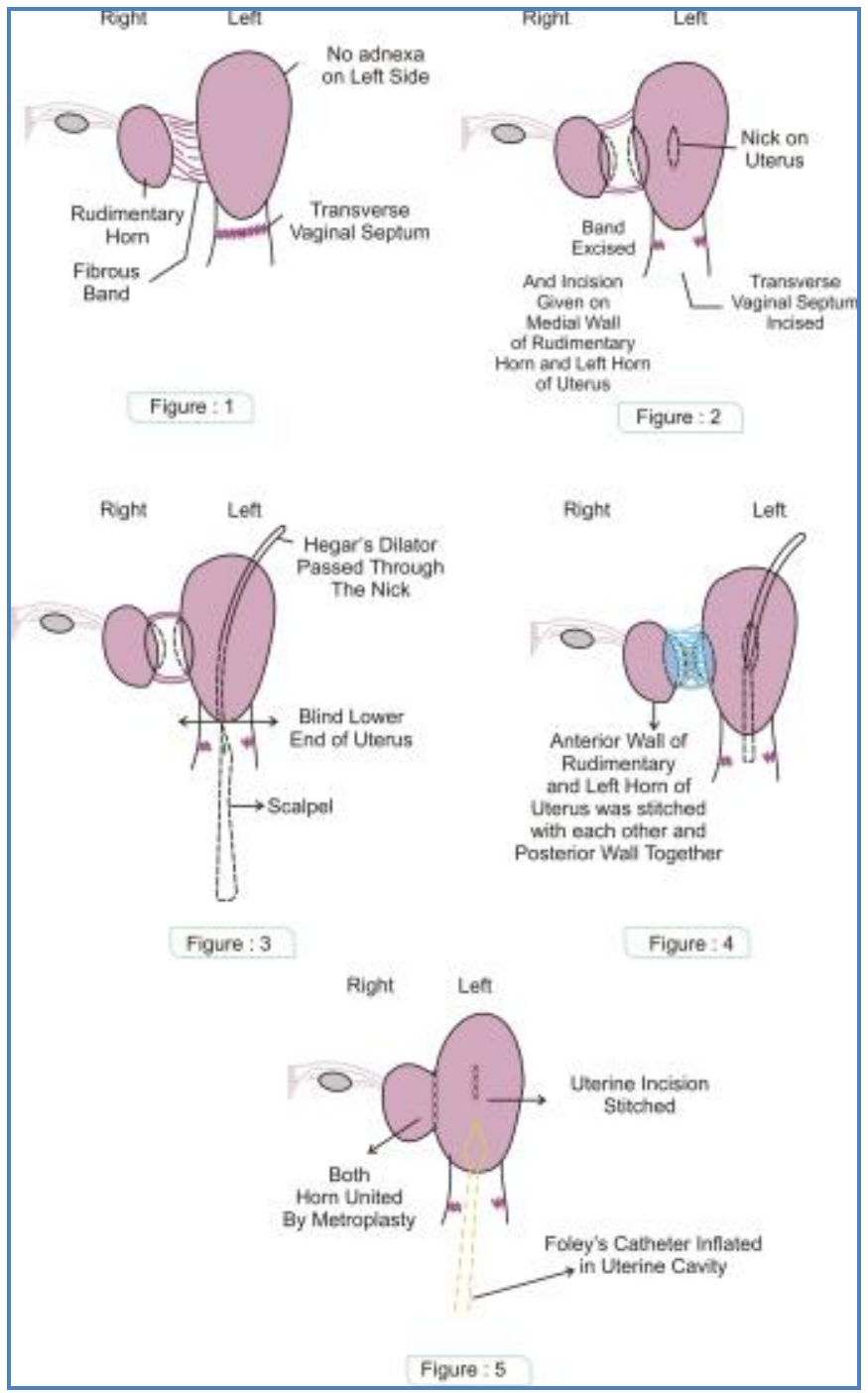

\section{AUTHORS:}

1. Ritu Mishra

2. Aditya Prakash Misra

\section{PARTICULARS OF CONTRIBUTORS:}

1. Assistant Professor, Department of Obstetrics and Gynaecology, Rama Medical College, Hospital and Research Centre, Kanpur.

2. Associate Professor, Department of, Radiodiagnosis, Rama Medical College, Hospital and Research Centre, Kanpur.

\section{NAME ADDRESS EMAIL ID OF THE CORRESPONDING AUTHOR:}

Dr. Ritu Mishra,

2A/43,

Azad Nagar,

Kanpur-208002.

Email: saggimishra@rediffmail.com

Date of Submission: 28/07/2014.

Date of Peer Review: 29/07/2014.

Date of Acceptance: 06/08/2014.

Date of Publishing: 11/08/2014. 